\title{
MODELAÇÃO TRIDIMENSIONAL DA ESTRUTURA GEOLÓGICA - GEO5 E REVIT - INTEROPERABILIDADE
}

\author{
Isabel Lopes $^{(1)}$, Luis Ribeirinho ${ }^{(1)}$ \\ (1) TPF - CONSULTORES DE ENGENHARIA E ARQUITETURA, S.A., Lisboa
}

\begin{abstract}
Resumo
O trabalho de interpretação geológica e geotécnica é realizado numa fase inicial dos projetos em que os dados são interpretados e os cortes de trabalho produzidos. Mesmo com uma boa preparação do trabalho é frequente que, por necessidade do projeto e/ou da obra, seja necessário realizar cortes adicionais, o que implica, trabalhando em $2 \mathrm{D}$, um período de espera para se produzir um novo corte geológico compatível com a estrutura 3D. Este é um dos aspetos que facilmente é ultrapassado quando definimos previamente a modelação tridimensional da geologia.

Em projetos complexos recorre-se a programas sofisticados de modelação geológica tridimensional, cujo investimento dificilmente é passível de ser justificado na rotina de projeto habitual. No entanto, dados os benefícios que a modelação tridimensional da geologia tem, particularmente na compatibilização com a modelação do projeto de estruturas geotécnicas e fundações, terá de haver uma mudança de paradigma.

Neste trabalho apresentamos um procedimento que permite importar no Revit a modelação geológica tridimensional. A modelação foi feita com um programa de modelação geológica mais simplificado, mas que permite exportar em IFC e landXML, o GEO5. Os ensaios foram exportados em IFC e importados diretamente no Revit, enquanto que os limites geológicos foram exportados em landXML e importados no Civil 3D, onde se geraram os volumes que foram posteriormente exportados em DWG para serem importados no Revit. Serão identificados os principais passos seguidos e as dificuldades sentidas na interoperabilidade entre os diferentes softwares.
\end{abstract}

\section{Introdução}

Em projeto geotécnico, de forma simplificada, pode dizer-se que os trabalhos de geologia e geotecnia têm como objetivo recolher informação sobre as características do maciço subterrâneo (solos e/ou rochas), conceptualizar o modelo geológico e efetuar a respetiva parametrização geotécnica, de forma a propor as soluções mais ajustadas para a infraestrutura a ser projetada. 
A modelação geológica e geotécnica consiste em expor as observações efetuadas e produzir uma interpretação efetuada com base nessas observações. A estrutura geológica é um elemento tridimensional. Em rotina habitual de projeto é apenas visualizada em desenhos de cortes efetuados bidimensionalmente, apesar de o geólogo compatibilizar os diversos cortes na sua interpretação da estrutura, que é obrigatoriamente 3D.

Normalmente o trabalho de interpretação geológica é realizado numa fase inicial dos projetos em que os dados de ordem geológica e geotécnica (observações) são interpretados e os cortes de trabalho produzidos. Mesmo com uma boa preparação do trabalho é, apesar de tudo, frequente que durante o desenvolvimento de um projeto seja necessário realizar cortes adicionais, por necessidade do projeto e/ou da obra. Para além disso, quando se lida com o espaço subterrâneo, por muita informação (observações) que se consiga recolher sobre as características dos materiais existentes, há sempre um grau de incerteza ligado à criação de modelos geológicos e geotécnicos. Essa incerteza é reduzida quando se aumenta a quantidade de observações, mas nunca é nula.

Em projetos mais complexos acontece recorrer-se a programas sofisticados de modelação geológica tridimensional, mas a maior parte desses programas têm um custo muito elevado e o investimento dificilmente é passível de ser justificado na rotina de projeto habitual. No entanto, dados os benefícios que a modelação da interpretação da geologia tridimensional tem, na compatibilização com a modelação do projeto de estruturas geotécnicas e fundações, terá de haver uma mudança de paradigma. Para além disso, julga-se que, simplificando o processo de geração de modelos tridimensionais, poderão criar-se de forma expedita diversos cenários geológico-geotécnicos incorporando as possíveis interpretações, podendo desta forma, representar a incerteza. Assim, na sua posterior integração em BIM será possível o dimensionamento e a construção das estruturas considerando essas incertezas.

Neste trabalho apresentamos um procedimento que permite importar no Revit a modelação geológica tridimensional. O modelo geológico foi efetuado com recurso a um programa de modelação geológica mais simplificado, mas que permite exportar em IFC e landXML, o GEO5 (módulo Estratigrafia).

Neste trabalho são identificados os principais passos seguidos e as dificuldades sentidas na interoperabilidade entre os diferentes softwares.

\section{Modelação em GEO5}

Para a modelação geológica tridimensional recorreu-se ao módulo Estratigrafia do programa GEO5 Geotechnical Software (Fine). Este programa permite criar um modelo tridimensional geológico, que pode, ou não, conter informação geotécnica, recorrendo à introdução de ensaios de campo. Os ensaios de campo que se podem introduzir neste programa correspondem a elementos pontuais, com desenvolvimento em profundidade, contendo diferentes tipos de informação de carácter geológico e/ou geotécnico.

O programa permite introduzir sondagens e poços, que são informação essencialmente de carácter geológico, e alguns ensaios geotécnicos, nomeadamente ensaios SPT (Ensaio de penetração dinâmica normalizado), CPTu (Ensaio de piezocone com medição da pressão de água nos poros), DPT (Ensaios de penetração dinâmica, ligeiros e pesados, DPL, DPM, DPH e DPSH), DMT (ensaio dilatómetrico) e PMT (ensaio pressiométrico). Alguns destes ensaios têm de ser realizados ao longo de furos de sondagem, como por exemplo o ensaio SPT, outros são 
efetuados por auto-cravação no terreno, como os ensaios DPT, CPT e DMT. Este programa não permite a introdução de ensaios sísmicos, quer com informação de tipo pontual ou bidimensional, nem qualquer outro tipo de ensaio geofísico.

É criada uma base de dados contendo os dados em bruto dos ensaios de campo, georreferenciados com $\mathrm{X}, \mathrm{Y}$ e Z . A introdução de $\mathrm{Z}$ está referenciada à superfície do terreno atual, mas caso existam dados referentes a campanhas geológicas e geotécnicas efetuadas em anos diferentes, e tenham existido movimentos de terras entre as diferentes campanhas (variação da superfície do terreno), o programa permite introduzir a distância entre a posição em que foi realizado o ensaio de campo e o $\mathrm{Z}$ da superfície de terreno atual.

No exemplo apresentado na Figura 1, os ensaios de campo utilizados foram sondagens, ensaios CPTu e ensaios DPSH. Esta figura representa a distribuição em área dos elementos pontuais e respetiva representação dos ensaios em bruto.

Para cada ensaio de campo tem que ser introduzida a interpretação geológica através da criação de um perfil de solo, em que se faz corresponder as camadas geológicas, ou um intervalo de resultados obtidos nos ensaios geotécnicos (Figura 2) à unidade geológica (ou geotécnica) que se quer definir. São essas interfaces entre unidades, definidas para os perfis de solo, que são a base da modelação tridimensional (Figura 3).

Para a modelação das interfaces o programa necessita ainda que todos os perfis de solo sejam relacionados com uma sondagem de referência, essa sondagem deve conter todas as interfaces entre camadas que sejam reconhecidas para a área de estudo (Figura 4).
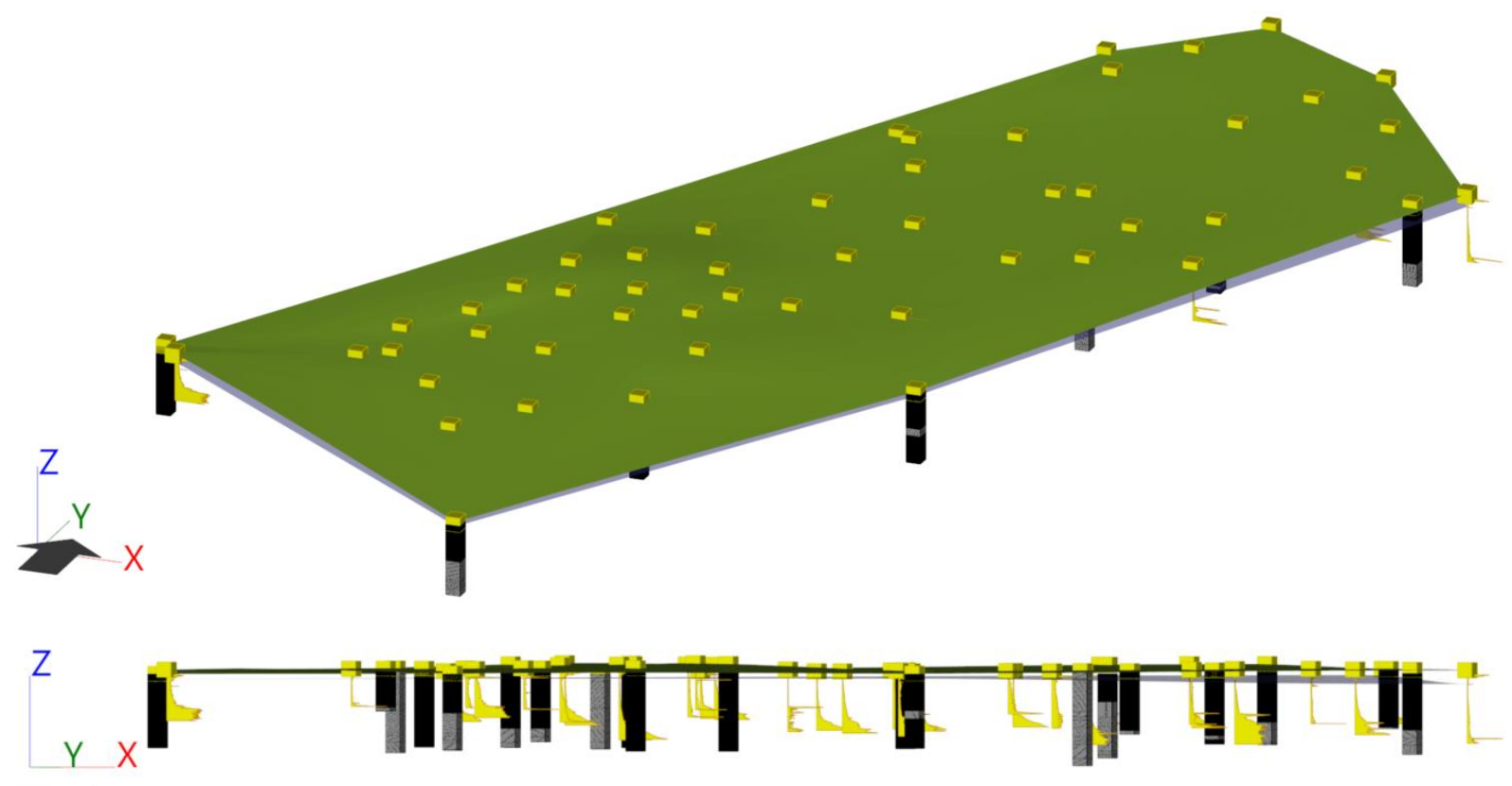

Figura 1: Dados em bruto introduzidos no módulo Estratigrafia (GEO5) e sua distribuição geográfica. 

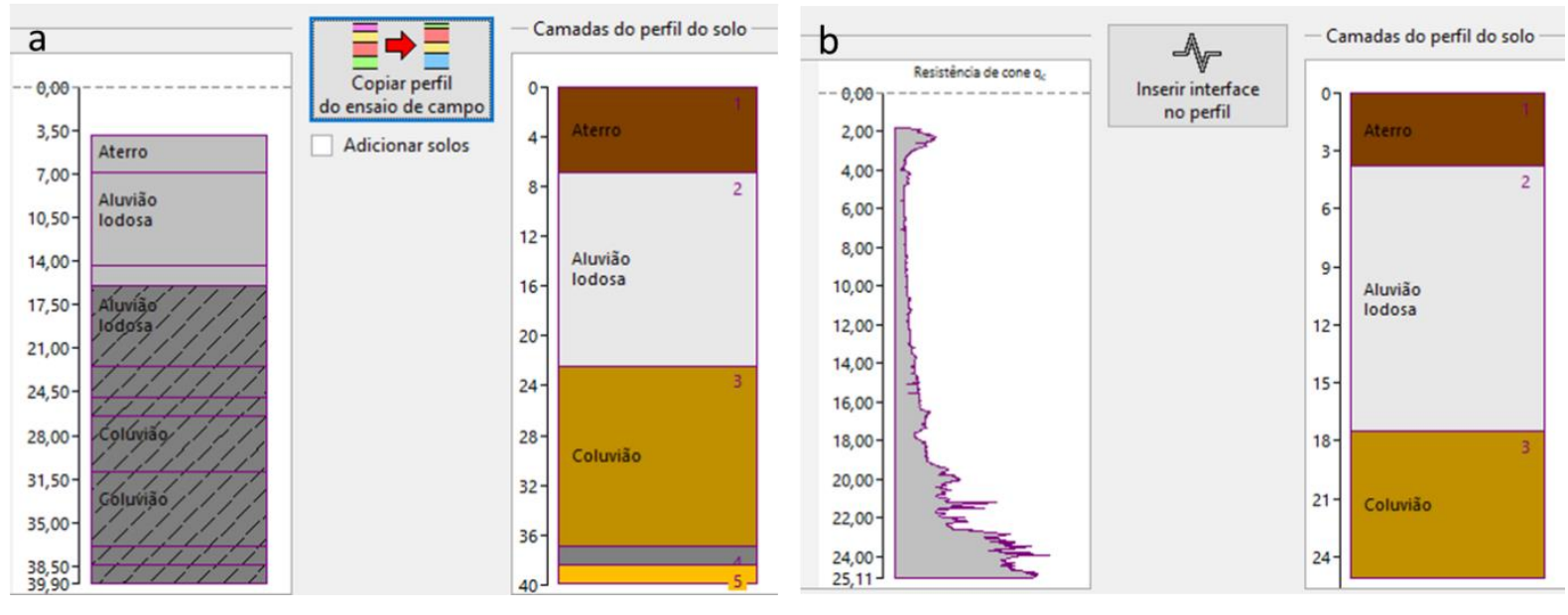

Figura 2: Definição dos perfis do solo no módulo Estratigrafia (GEO5): a. de uma sondagem; b. de um ensaio CPT.

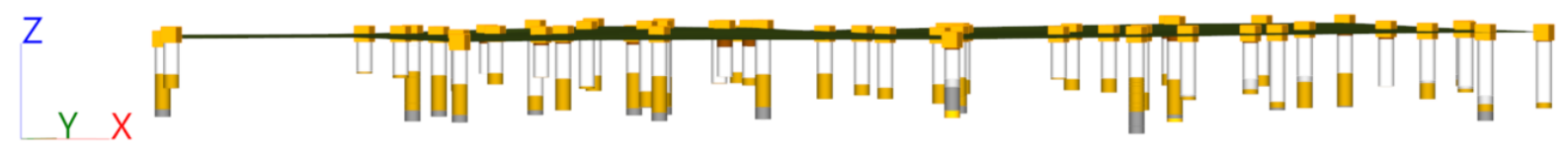

Figura 3: Perfis de solo interpretados de todos os ensaios de campo e respetiva distribuição geográfica.

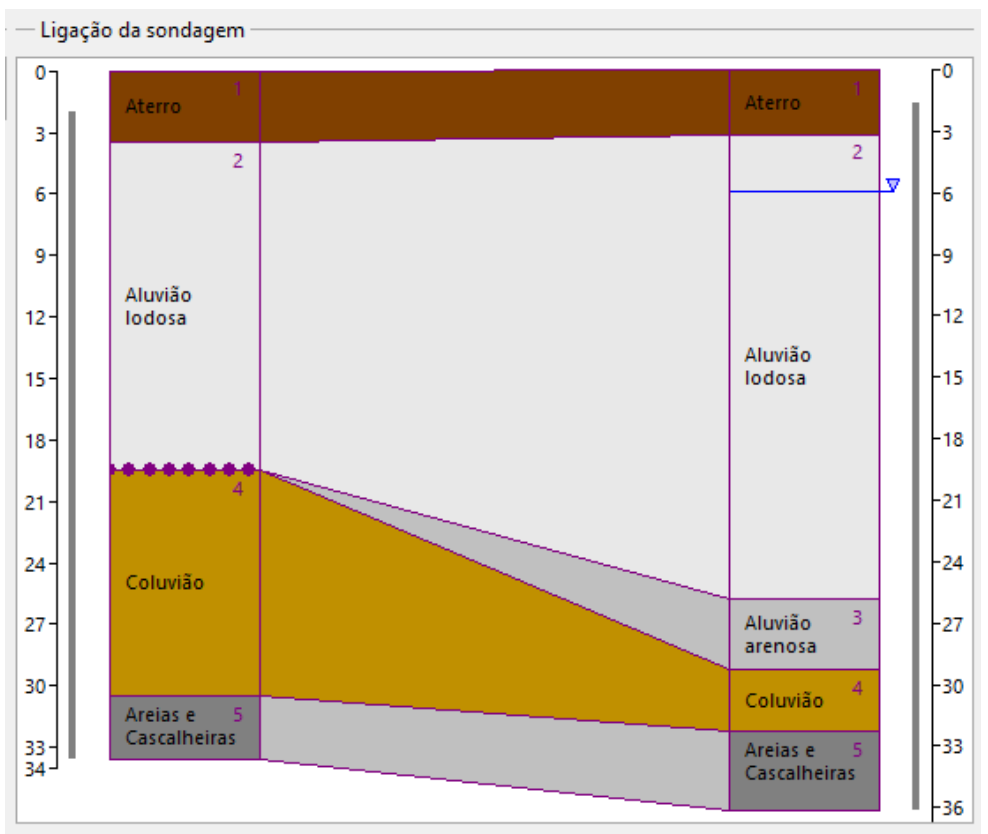

Figura 4: Estabelecimento das interfaces no perfil de solo relativamente à sondagem de referência do modelo.

A geração do modelo é posteriormente feita por ligação entre as interfaces definidas para as camadas, de uma forma geral, ligando essas interfaces entre os diversos pontos onde foram 
identificadas, e escolhendo, ou não, a suavização da interface (Figura 5). No entanto, o programa não é restritivo à criação de interfaces exclusivamente através dos perfis de solo e permite adicionar a interpretação do geólogo através da identificação e localização das interfaces em algumas áreas ou pontos.
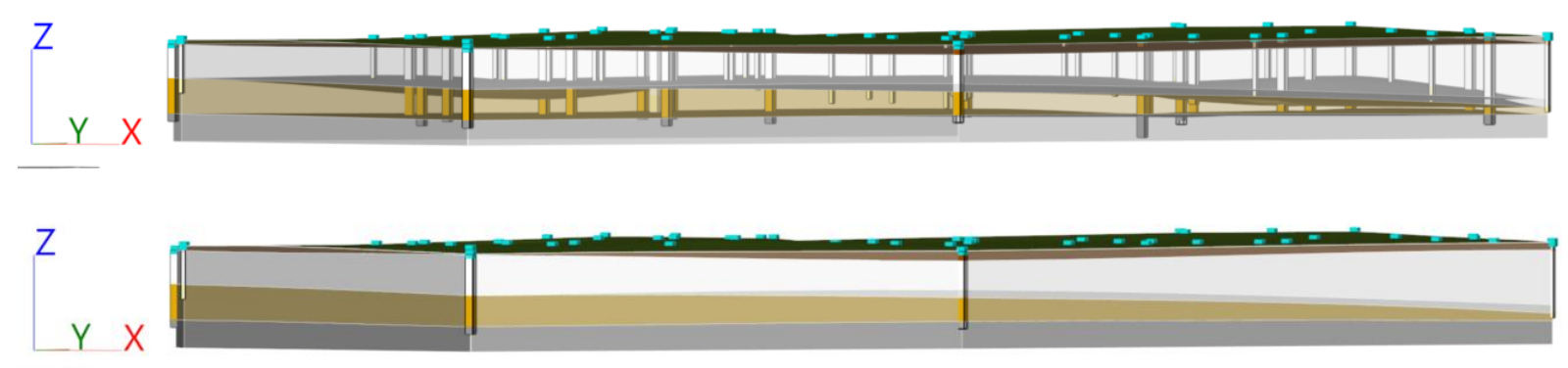

Figura 5: Modelo tridimensional da geologia, interfaces e bloco diagrama.

Após geração do modelo este pode ser exportado em IFC e em landXML, permitindo posteriormente a sua utilização nos ambientes de trabalho do projeto geotécnico.

\section{Fluxo de trabalho para os ensaios}

A informação relativa aos ensaios, nomeadamente DPSH, CPTu e Sondagens foi inicialmente exportada do GEO5 em IFC e importada diretamente no Revit. Apesar de graficamente os ensaios terem um aspeto aceitável, topologicamente não eram utilizáveis. Todos os elementos estavam classificados como generic models e todos tinham um conjunto muito alargado de parâmetros, criados automaticamente pelo GEO5. Foram criados parâmetros específicos para cada elemento, em que o nome do elemento fazia parte do nome do parâmetro, o que só por si já seria desaconselhável, e, para além disso, eram parâmetros de projeto, ou seja, aparecem em todos os elementos da classe a que foram atribuídos. Na prática, o número de parâmetros de cada elemento era seis vezes o número total de elementos. Uma vez que no caso em análise existiam 53 elementos, cada generic model tinha 318 parâmetros:

- Tipo de ensaio: (Parâmetros do ensaio: XXX)

- Coordenada: x (Parâmetros do ensaio: XXX)

- Coordenada: y (Parâmetros do ensaio: XXX)

- Coordenada: z (Parâmetros do ensaio: XXX)

- Prof. do $1^{\circ}$ pto. a partir da sup. de terreno original: d1 (Parâmetros do ensaio: XXX)

- Ensaio de campo gera perfil do solo: (Parâmetros do ensaio: XXX)

Alternativamente, foi criada uma família com três tipos, um para cada tipo de ensaio (ver Figura 6). Nessa família foram criados 7 parâmetros:

- Element ID

- Element Type 
- Coordinate $X$

- Coordinate $Y$

- Coordinate Z

- Depth

- $\quad$ First Point Depth

Note-se que ao introduzir o parâmetro Element ID, utilizado para identificar o ensaio, reduzimos o número de parâmetros de 318 para 7, sendo um desses parâmetros a profundidade, Depth, que não estava disponível no IFC e era necessária para a representação gráfica. Aos novos parâmetros criados atribuíram-se tipos de parâmetros (text, length, number, etc.) correspondentes ao tipo de informação que transportam. Os parâmetros do IFC eram todos do tipo text. O desenvolvimento interno da família permitiu customizar a aparência dos ensaios à imagem da TPF Consultores. Todas as famílias desenvolvidas internamente têm o nome e os parâmetros em Inglês. O preenchimento dos parâmetros na nova família foi feito com recurso a uma rotina de Dynamo que extrai a informação do IFC. A profundidade foi preenchida a partir de um ficheiro de Excel.

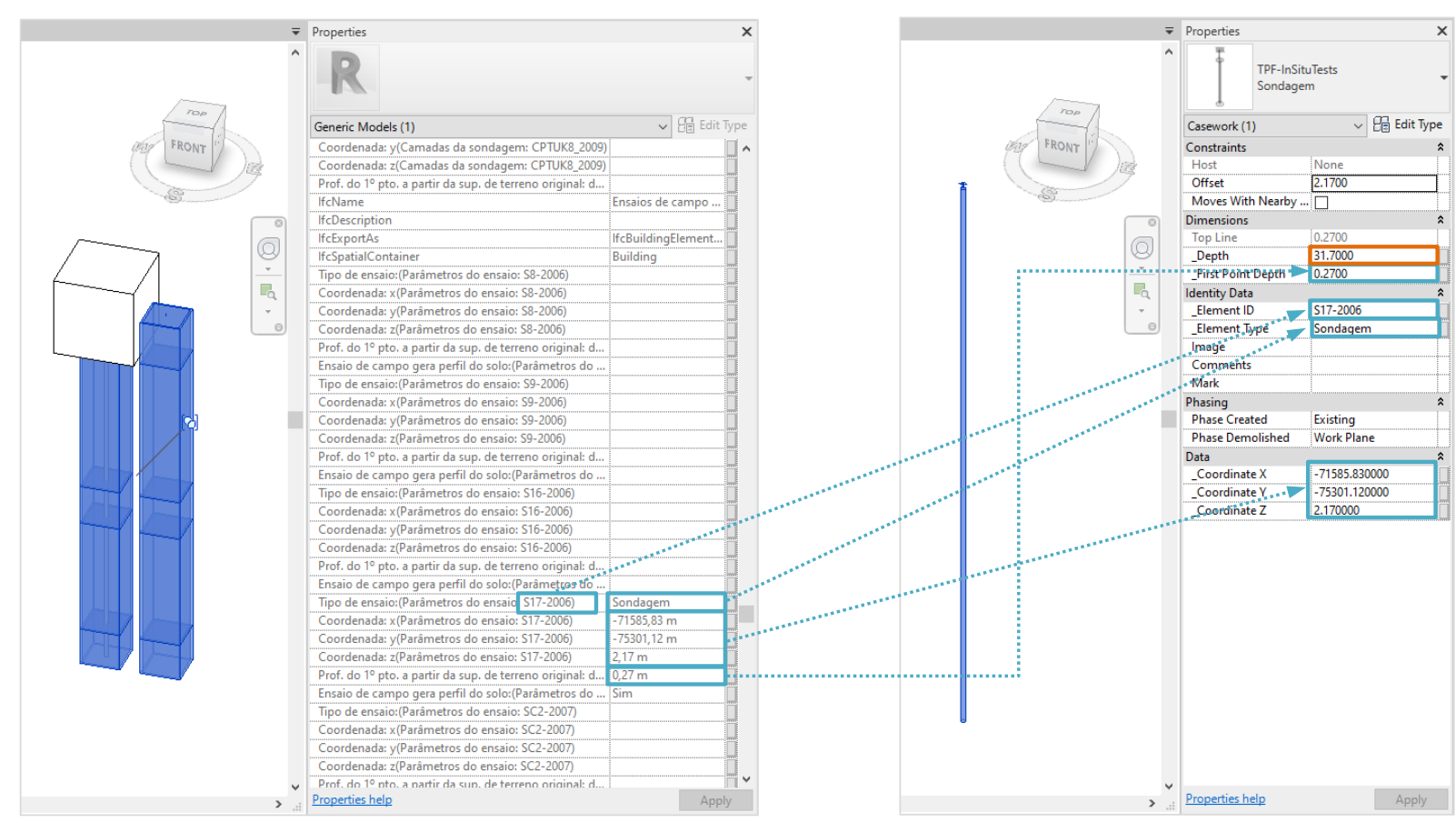

Figura 6: Sondagem do IFC à esquerda e sondagem da TPF à direita no modelo de Revit.

Estas alterações extravasam o ambiente Revit, uma vez que podem ser transportadas para o formato IFC. No que diz respeito aos ensaios, o IFC gerado a partir do Revit ficou melhor estruturado do que o produzido originalmente pelo GEO5, permitindo até gerar tabelas com a informação associada a cada ensaio, como se pode constatar na Figura 7. Esta informação mantém os tipos de dados criados no Revit, como é o caso do parâmetro First Point Depth que está definido como comprimento, ao contrário do que acontecia com os parâmetros do IFC exportado do GEO5 (que eram do tipo text). 


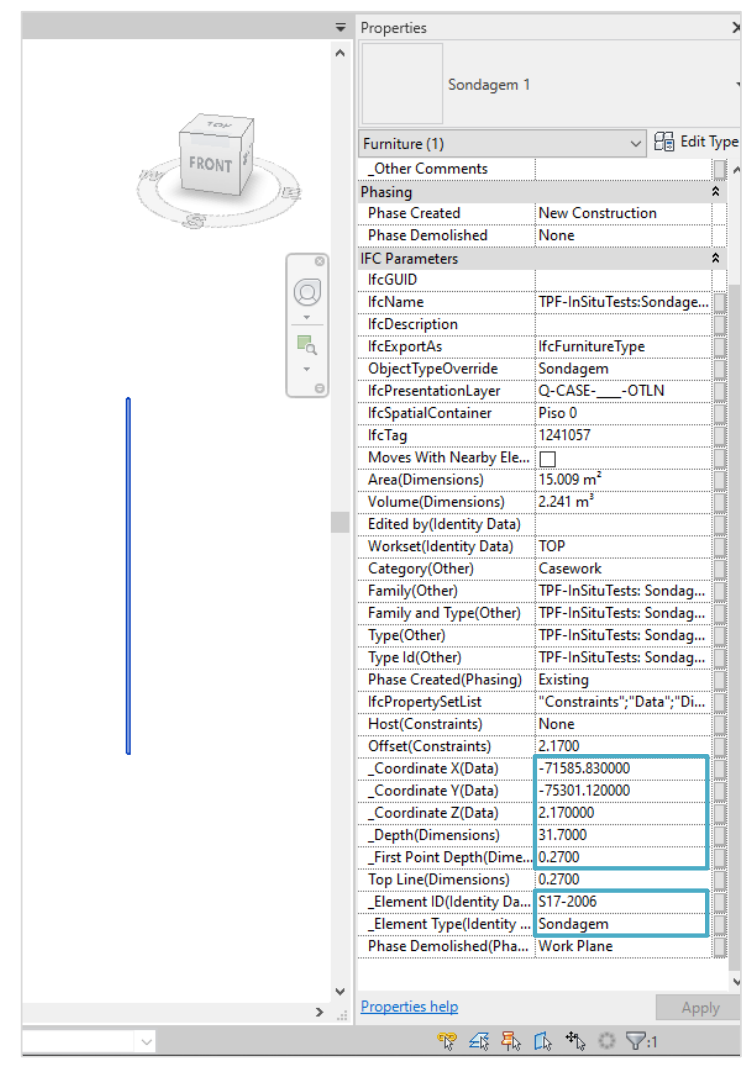

\begin{tabular}{|c|c|c|c|c|c|c|}
\hline \multicolumn{7}{|c|}{ Ensaios } \\
\hline $\begin{array}{l}\text { Element Type } \\
\text { (Identity Data) }\end{array}$ & $\mid \begin{array}{c}\text { Element ID } \\
\text { (Identity Data) }\end{array}$ & $\begin{array}{l}\text { Coordinate X } \\
\text { (Data) }\end{array}$ & $\begin{array}{c}\text { Coordinate } Y \\
\text { (Data) }\end{array}$ & $\begin{array}{l}\text { Coordinate Z } \\
\text { (Data) }\end{array}$ & \begin{tabular}{|c} 
First Point Depth \\
(Dimensions)
\end{tabular} & $\begin{array}{c}\text { Depth } \\
\text { (Dimensions) }\end{array}$ \\
\hline CPT & CPTU06 2006 & -71899.89 & -75377.04 & 3.9 & 1.71 & 20.12 \\
\hline CPT & CPTU07_2006 & -71702.4 & .75018 .61 & 3.83 & 1.79 & 23.32 \\
\hline \begin{tabular}{|l} 
CPT \\
\end{tabular} & CPTU12_2006 & -71526.4 & .75122 .2 & 3.94 & 2.01 & 22.84 \\
\hline CPT & CPTU 03_2007 & -71670.24 & .75231 .29 & 4.05 & 2.26 & 24.34 \\
\hline CPT & CPTU_C_2008 & -71651.9 & .74899 .66 & 6.38 & 4.59 & 20.72 \\
\hline CPT & CPTU_F_2008 & -71447.12 & -74997.66 & 3.83 & 1.92 & 29.28 \\
\hline CPT & CPTUK1_2009 & -71705.62 & .75259 .01 & 6.19 & 2.54 & 19.96 \\
\hline CPT & CPTUK2_2009 & -71686.03 & .75301 .46 & 6.63 & 3.03 & 16.90 \\
\hline CPT & CPTUK3 2009 & -71824.81 & -75351.83 & 4.3 & 0.55 & 15.56 \\
\hline \begin{tabular}{|l|} 
CPT \\
\end{tabular} & CPTUK5_2009 & -71797.41 & -75285.59 & 4.43 & 0.68 & 16.10 \\
\hline CPT & CPTUK4_2009 & -71732.66 & .75386 .86 & 4.42 & 0.77 & 16.80 \\
\hline CPT & CPTUK7_2009 & -71613.49 & .75061 .12 & 3.4 & 0.00 & 24.34 \\
\hline CPT & CPTUK8_2009 & -71548.89 & -74917.93 & 4.53 & 0.83 & 22.62 \\
\hline DPT & DPSH5 & -71757.15 & .75416 .18 & 3.22 & 0.00 & 21.40 \\
\hline DPT & DPSH6 & -71784.04 & .75309 .69 & 4.22 & 0.57 & 20.00 \\
\hline DPT & DPSH7 & -71713.1 & -75206.83 & 6.06 & 2.41 & 20.80 \\
\hline DPT & DPSH8 & -71602.71 & -75142.79 & 3.43 & 0.00 & 20.80 \\
\hline DPT & DPSHK1 & -71776.22 & .75221 .56 & 6.77 & 3.08 & 23.40 \\
\hline DPT & DPSHK2 & -71766.13 & -75253.92 & 5.86 & 2.14 & 22.20 \\
\hline DPT & DPSHK3 & -71737.66 & -75241.51 & 6.13 & 2.51 & 29.40 \\
\hline DPT & DPSHK4 & -71731.95 & .75272 .72 & 6.1 & 2.45 & 29.80 \\
\hline DPT & DPSHK5 & -71738.18 & -75157.88 & 3.56 & 0.00 & 20.40 \\
\hline DPT & DPSHK6 & -71622.93 & -75222.38 & 3.43 & 0.00 & 28.00 \\
\hline DPT & DPSHK7 & -71680.89 & -75054.27 & 3.4 & 0.00 & 25.00 \\
\hline DPT & DPSHK8 & -71652.97 & .75003 .97 & 3.61 & 0.00 & 24.00 \\
\hline DPT & DPSHK9 & -71656.75 & .75118 .4 & 3.37 & 0.00 & 25.60 \\
\hline DPT & DPSHK10 & -71499.11 & -74996.17 & 4.17 & 0.49 & 27.40 \\
\hline DPT & DPSHK11 & -71568.88 & .74957 .48 & 4.42 & 0.79 & 31.40 \\
\hline DPT & DPSHK12 & -71528.71 & -74883.02 & 4.51 & 1.02 & 22.40 \\
\hline DPT & DPSHK13 & -71617.24 & -74884.49 & 6.53 & 2.88 & 22.80 \\
\hline DPT & DPSHK16 & -71703.71 & .75107 .7 & 3.36 & 0.00 & 25.20 \\
\hline DPT & DPSHK17 & -71670.33 & -75164.94 & 3.27 & 0.00 & 26.40 \\
\hline DPT & DPSHK18 & -71601.28 & -75055.79 & 3.41 & 0.00 & 31.40 \\
\hline Sondagem & S8-2006 & -71782.24 & -75162.26 & 3.51 & 1.41 & 32.00 \\
\hline Sondagem & S9-2006 & -71640.51 & .74922 .05 & 6.3 & 3.90 & 36.00 \\
\hline Sondagem & S16-2006 & -71464.28 & .75019 .1 & 3.95 & 1.65 & 34.50 \\
\hline Sondagem & S17-2006 & -71585.83 & -75301.12 & 2.17 & 0.27 & 31.70 \\
\hline
\end{tabular}

Figura 7: Imagens do IFC exportado do Revit - à esquerda um ensaio e respetivas propriedades, à direita tabela com todos os ensaios.

\section{Fluxo de trabalho para os limites geológicos}

Por uma questão gráfica, de representação, era importante que o espaço entre os limites geológicos estivesse preenchido, para que se pudessem associar as cores correspondentes à geologia de cada camada. Para que isso fosse possível, os limites geológicos gerados pelo GEO5 foram exportados em landXML e importados no Civil 3D.

Uma das primeiras dificuldades esteve relacionada com um problema de exportação do GEO5 para landXML que inverte a posição das coordenadas. A solução encontrada para resolver o problema temporariamente, enquanto não é publicada uma versão do software com esta questão corrigida, foi extrair as coordenadas dos pontos que constituem as superfícies e gerar novos pontos com as coordenadas corretas, a partir dos quais se geraram novas superfícies.

O Civil 3D tem uma ferramenta que permite gerar sólidos entre duas superfícies. Esses sólidos foram exportados em dwg e inseridos no Revit como links em famílias do tipo model in-place (ver Figura 7). Houve novamente dificuldades relacionadas com a georreferenciação. Neste caso o problema está na forma como o Revit lida com as coordenadas dentro das famílias. No caso específico das famílias model in-place, a origem é o project base point (PBP), que neste projeto, como em quase todos os projetos georreferenciados, não coincide com a origem do sistema de coordenadas utilizado. Houve então necessidade de gravar ficheiros locais, isto é, de 
fazer uma translação dos sólidos das coordenadas do PBP para a origem do sistema de coordenadas.

Era imperativo que os sólidos estivessem dentro de famílias, particularmente para se poderem cortar. Recorde-se que este era um dos principais objetivos do modelo geológico: permitir a extração de cortes em qualquer sítio do modelo (Figura 8), sem necessidade de interpretação da equipa de geologia. Para além disso, o controlo da visualização é mais simples estando os sólidos dentro de famílias.

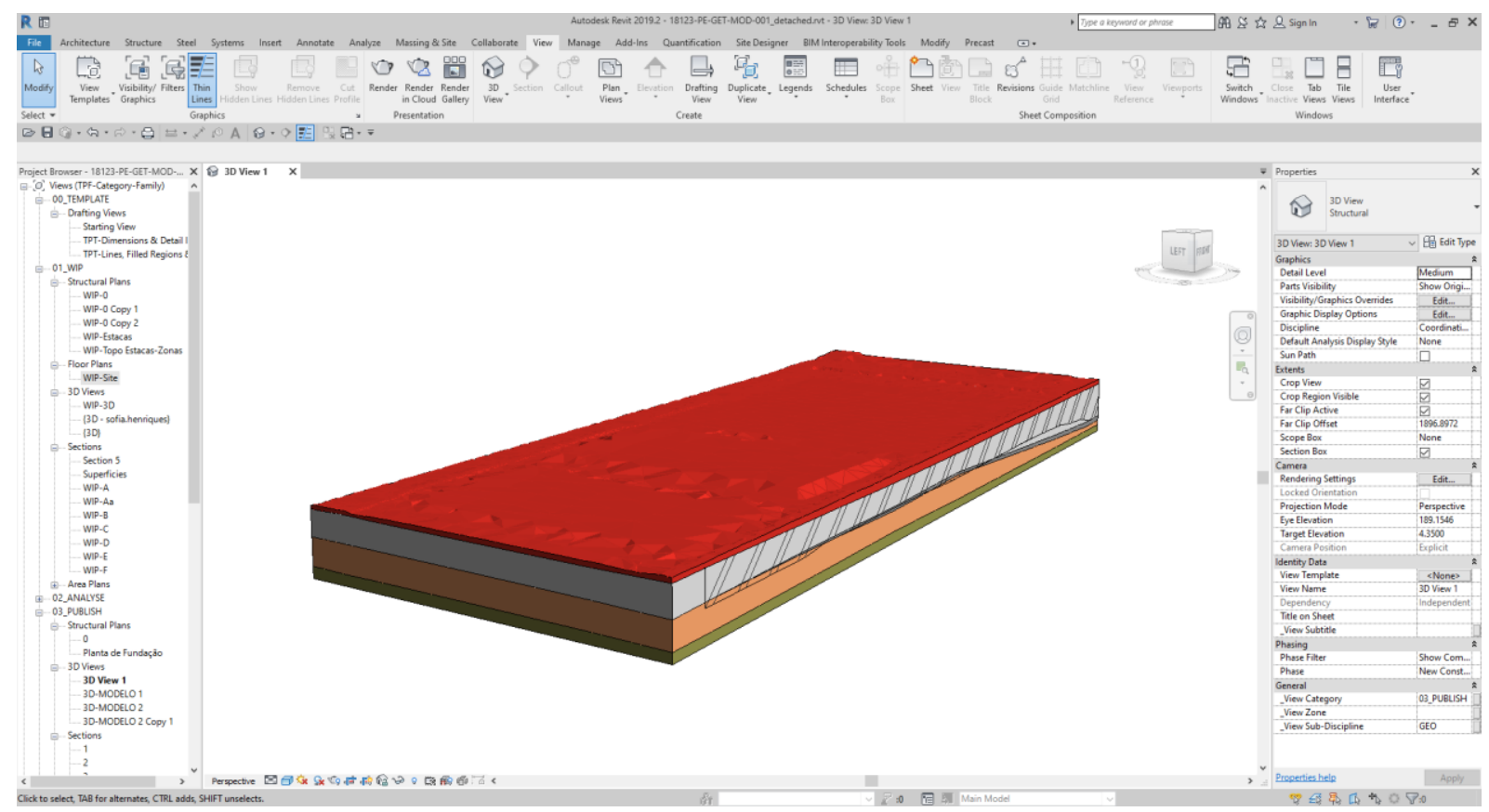

Figura 7: Estrutura geológica no modelo de Revit.

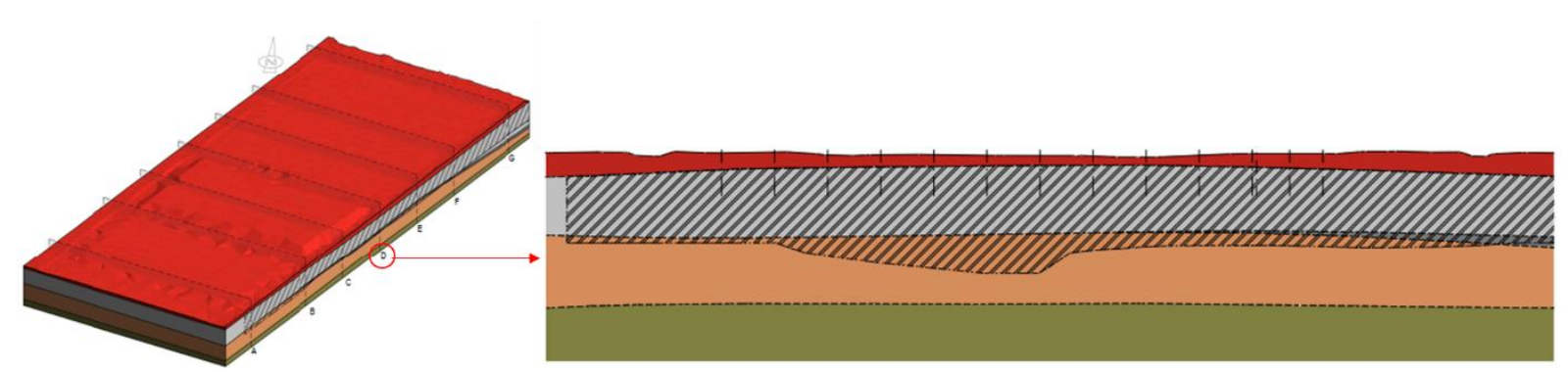

Figura 8: Corte geológico-geotécnico obtido do modelo de Revit.

\section{Conclusões}

O módulo Estratigrafia do GEO5 provou ser uma boa ferramenta para a modelação geológica tridimensional, uma vez que a sua interface é relativamente simples e intuitiva, com fácil introdução dos ensaios de campo e, na modelação, permite adicionar a interpretação do geólogo. O programa permite exportar em formatos que poderiam ser importados diretamente no Revit. No entanto, a forma como os ficheiros são estruturados pelo GEO5 requer processamento, 
tornando todo o processo menos expedito. Apesar da facilidade de criar diversos modelos no GEO5 com os mesmos dados em bruto e dos procedimentos internos desenvolvidos para otimizar o processamento adicional necessário para a utilização no Revit, o facto de ser imprescindível esse processamento, dificulta a criação de vários cenários geológicogeotécnicos nos prazos normalmente atribuídos à realização dos projetos. Contudo, a possibilidade de exportação para landXML e IFC só ficou disponível na versão GEO5 2019, podendo ainda ser alvo de melhoria nas próximas versões do programa.

O trabalho desenvolvido para a integração do modelo geológico-geotécnico em Revit provou ser uma mais-valia na realização do projeto geotécnico, permitindo criar rotinas que relacionam os elementos geotécnicos com a geologia. 